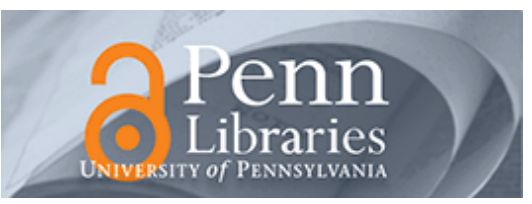

University of Pennsylvania ScholarlyCommons

\title{
8-2012
}

\section{Evaluation of a Smart Alarm for Intensive Care Using Clinical Data}

\author{
Andrew King \\ University of Pennsylvania, kingand@cis.upenn.edu \\ Kelsea Fortino \\ Drexel University \\ Nicholas Stevens \\ Stanford University \\ Sachin Shah \\ University of Pennsylvania \\ Margaret Fortino-Mullen \\ University of Pennsylvania
}

See next page for additional authors

Follow this and additional works at: https://repository.upenn.edu/cis_papers

\section{Recommended Citation}

Andrew King, Kelsea Fortino, Nicholas Stevens, Sachin Shah, Margaret Fortino-Mullen, and Insup Lee, "Evaluation of a Smart Alarm for Intensive Care Using Clinical Data", 34th Annual International Conference of the IEEE Engineering in Medicine and Biology Society (EMBC '12) , 166-169. August 2012.

http://dx.doi.org/10.1109/EMBC.2012.6345897

The $34^{\text {th }}$ Annual International Conference of the IEEE Engineering in Medicine and Biology Society (EMBC'12), San Diego, California, USA, August 28 - September 1, 2012

http://embc2012.embs.org/

This paper is posted at ScholarlyCommons. https://repository.upenn.edu/cis_papers/737

For more information, please contact repository@pobox.upenn.edu. 


\title{
Evaluation of a Smart Alarm for Intensive Care Using Clinical Data
}

\begin{abstract}
We describe and report the results of an evaluation of a smart alarm algorithm for post coronary artery bypass graft (CABG) patients. The algorithm (CABG-SA) was applied to vital sign data streams recorded in a surgical intensive care unit (SICU) at a hospital in the University of Pennsylvania Health System. In order to determine the specificity of CABGSA, the alarms generated by CABG-SA were compared against the actual interventions performed by the staff of the critical care unit. Overall, CABG-SA alarmed for $55 \%$ of the time relative to traditional alarms while still generating alarms for 12 of the 13 recorded interventions.
\end{abstract}

\section{Comments}

The $34^{\text {th }}$ Annual International Conference of the IEEE Engineering in Medicine and Biology Society (EMBC'12), San Diego, California, USA, August 28 - September 1, 2012

http://embc2012.embs.org/

\section{Author(s)}

Andrew King, Kelsea Fortino, Nicholas Stevens, Sachin Shah, Margaret Fortino-Mullen, and Insup Lee 


\title{
Evaluation of a Smart Alarm for Intensive Care using Clinical Data
}

\author{
Andrew King ${ }^{1}$, Kelsea Fortino ${ }^{3}$, Nicholas Stevens ${ }^{4,5}$, Sachin Shah ${ }^{2}$, Margaret Fortino-Mullen ${ }^{2}$ and Insup Lee ${ }^{1}$
}

\begin{abstract}
We describe and report the results of an evaluation of a smart alarm algorithm for post coronary artery bypass graft (CABG) patients. The algorithm (CABG-SA) was applied to vital sign data streams recorded in a surgical intensive care unit (SICU) at a hospital in the University of Pennsylvania Health System. In order to determine the specificity of CABGSA, the alarms generated by CABG-SA were compared against the actual interventions performed by the staff of the critical care unit. Overall, CABG-SA alarmed for $55 \%$ of the time relative to traditional alarms while still generating alarms for 12 of the 13 recorded interventions.
\end{abstract}

\section{INTRODUCTION}

The ability to provide care to patients with complex health problems has primarily been facilitated by the increasing availability of life supporting technologies, such as mechanical ventilation and dialysis. Use of these technologies requires caregivers to closely monitor the patient to determine their response to treatment and prevent complications. Vigilant monitoring of critically ill patients facilitates the ability of practitioners to detect problems early and intervene before complications occur. Typically, critical care nurses care for two patients and the responsibility of monitoring is shared with other patient tasks such as coordination of care goals, wound care, medication administration and bathing and feeding the patient. Clinical alarms are designed to support the caregiver by signaling a change in the patients condition. First generation ventilators introduced in the early 1960s lacked alarms. Fairman and Kagan describe an incident where a patient accidentally disconnected from the ventilator [1]. Without a ventilator alarm, caregivers were unaware of the situation and the patient died. Similar incidents prompted the development of alarm systems [2]. The development of micro-electronics in the seventies introduced a greater number of monitoring devices, each with their own alarm systems. Caregivers are required to define alarm parameters for each medical device. With so many medical devices, each having alarms and lacking interoperability, there is an overabundance of alarms and the nurse experiences cognitive overload. It has been reported that 85 percent of alarms are false and do not prompt an intervention [3]. The high number of false alarms results in alarm fatigue, where the practitioner becomes desensitized to the alarm and does

1 Department of Computer and Information Science, University of Pennsylvania

${ }^{2}$ University of Pennsylvania Health System, University of Pennsylvania

${ }^{3}$ Drexel University

${ }_{5}^{4}$ Department of Electrical and Systems, University of Pennsylvania

${ }^{5}$ Current affiliation: Computer Science Department, Stanford not respond with a sense of urgency [4]. In many cases having too many alarms is as harmful as having no alarms. Most physiologic monitors used in critical care have the ability to simultaneously survey heart rate and rhythm, blood pressure, respirations and oxygenation. Alarms are set individually for each physiologic parameter and are triggered whenever the vital sign falls outside of the predetermined limit. An alarm is generated if the patients heart rate is below the threshold; a second alarm activates if the respiratory alarm is outside of range. Clinicians, however rarely use one data point at one point in time to make decisions. Clinicians evaluate all data points over a period of time to determine trends before employing interventions. The University Pennsylvania School of Engineering partnered with clinicians from the University of Pennsylvania Health System to develop an alarm algorithm that would appropriately alert the clinician to clinical conditions requiring immediate attention while reducing the number of false alarms.

\section{CABG SMART AlARM}

Post-operative patients after artery bypass graft (CABG) surgery are at particular risk of physiologic instability [5]. Continuous monitoring of a combination of common vital signs in the intensive care unit allows for detection of physiologic changes so practitioners may intervene in a timely manner and prevent complications. In many cases, however, individual thresh-hold alarms can produce a large number of false positives. For example, the fingerclip sensor of a pulse-oximeter can fall off the patient or react to the artificial lighting of the care environment to produce erroneous readings. These sorts of basic failures can be mitigated by a system which is able to monitor multiple vital signs and distinguish data artifacts (such as disconnected leads which lead to sharp vital sign drops) from true patient distress.

Previous work [6] describes a rule-based smart alarm for CABG patients, CABG-SA, which combines four major vital signs commonly monitored in the ICU: heart rate (HR), blood pressure (BP), blood oxygen saturation $\left(\mathrm{SpO}_{2}\right)$, and respiratory rate (RR). Figure 2 provides a block diagram of CABG-SA. ICU nurses were interviewed to determine ranges for classifying each vital sign as a member of some collection of fuzzy sets (classifying, for example, a blood pressure between 50 and 60 as a mix of 'Low' and 'Normal', between 60 and 100 as 'Normal', and between 100 and 107 as being a mix of 'Normal' and 'High'. See Figure 1). Afterward, nurses determined rules that identified combinations of these vital sign statuses which would be cause for concern. An 


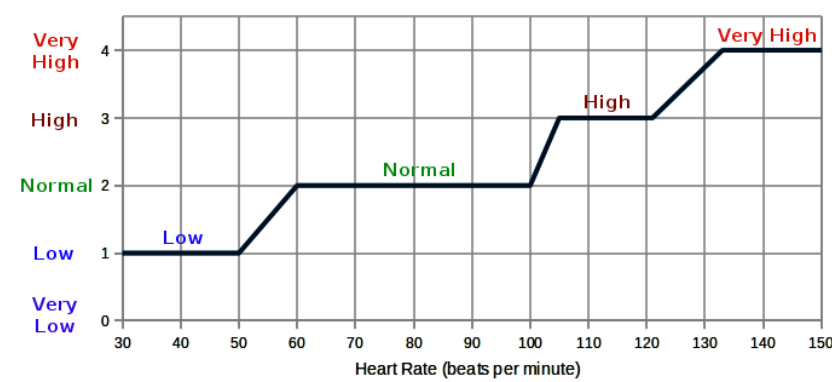

Fig. 1. Sample fuzzy set classification for Heart Rate

\begin{tabular}{|c|c|c|c||c|}
\hline BP & HR & $\mathrm{SPO}_{2}$ & RR & Alarm Level \\
\hline \hline Normal & Normal & Normal & Normal & 0 \\
High & Normal & Normal & Low & 1 \\
High & Low & Normal & Normal & 2 \\
Very Low & Normal & Normal & High & 3 \\
High & High & Low & High & 2 \\
\hline
\end{tabular}

TABLE I

SMALL SUBSET OF THE RULE SET.

example fragment of the rule table is shown in Table I. The 'smart' alarm worked by monitoring a patient's four vitals, classifying those into fuzzy sets, and searching the rule table for the corresponding alarm level to output. To deal with missing data (due to network or sensor faults), rapid drops to zero are conservatively classified as 'Low' for the duration of the signal drop. This feature was intended to prevent the algorithm from generating false positives; if the other three vital signs are available and indicate that the patient is stable, then an alarm is not raised.

Classification of vital signs using fuzzy sets helped to overcome the difficulty of establishing a ruleset customized to each patient's baseline vital signs. Fuzzy set classifiers can easily be modified to address a specific patient with, for example, a very low 'Normal' resting heart rate, without rewriting the entire rule set.

\section{iII. Evaluation Methodology}

Previously, the CABG-SA was evaluated by executing the CABG-SA on Physiobank [7] datastreams. In parallel, an experienced SICU nurse annotated those same datastreams, indicating over what periods of time an alarm should be active (i.e., when the physiologic state of the patient might

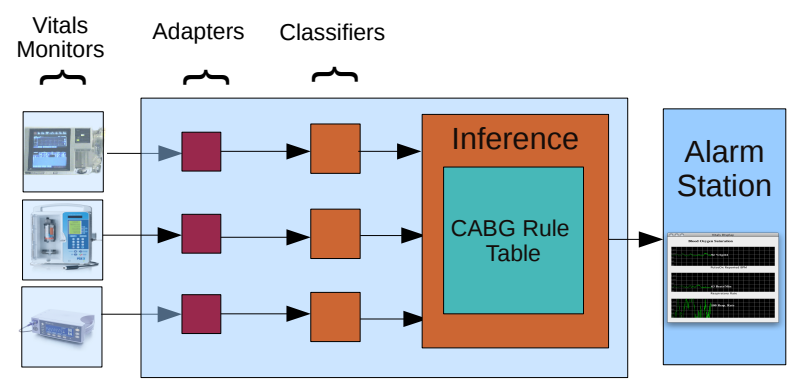

Fig. 2. CABG Smart Alarm block diagram imply the patient is in trouble). While this previous study indicated that the CABG-SA showed promise in reducing the amount of alarms (57.13 percent over 1,451 hours of recorded patient data from Physiobank) while not missing any so-called true alarms, we don't actually know what clinical interventions were attempted for the patients recorded in the PhysioNET databank. To this end, we conducted an evaluation of the CABG-SA using minute-by-minute vitals sign data from post-CABG patients at a Hospital in the University of Pennsylvania Health System (UPHS). This afforded us the opportunity to observe how clinicians reacted to the traditional monitor alarms and record any interventions the clinicians performed to stabilize the patient.

Data collection took place in a 16-bed surgical intensive care unit. A research assistant (RA) directly observed and recorded monitor alarms and clinician response. Observation was conducted directly in the room or the central monitoring station. The goal of observation was to capture as many alarms as possible; immediate post-operative patients were the first choice for observation as this condition typically generates the greatest number of alarms. When data from post-operative patients was not available, alarm data was collected from the central monitoring station. During the observations, the RA looked at four main vital signs: heart rate, arterial blood pressure, pulse-ox, and respiratory rate. When one of these alarms activated, the RA recorded the time, the type of alarm, the value of the alarm, the time the alarm shut off, and if the alarm triggered an intervention. Here, an intervention was defined as any action the nurse took, which was triggered by an alarm or set of alarms, to improve the patients condition. Once the observation period was complete, the RA conferred with an expert critical care nurse to ensure accuracy and relevancy of information. All patients were monitored with a standard monitoring system that linked through an interface to VisICUE [8]. Vital sign messages were sent from the Philips monitoring system in 60 -second intervals containing the patient data as monitored. Messages were transmitted in an HL7 format and then stored in the UPHS datastore.

Twenty-seven CABG patients were observed while they convalesced in the ICU immediately after having a CABG operation. Out of these 27 patients, 9 had appropriate vital sign samples stored in the hospital IT system during the time period of the observation. Each of these patients were observed for between 26 and 127 minutes, totalling 751 minutes of observation.

In order to compare monitor alarm performance with the CABG smart alarm, the minute by minute samples of these patient's physiologic state were retroactively retrieved (after the observations) from the UPHS datastore. The smart alarm algorithm was applied to the retrieved datastreams, resulting in a trace of the smart alarm outputs that would have been produced if the smart alarm were active at the patient's bed side.

Because of the rate at which a patient can deterioriate, and the expected response time of the care staff, an intervention alarm is considered covered by a smart alarm if the smart 


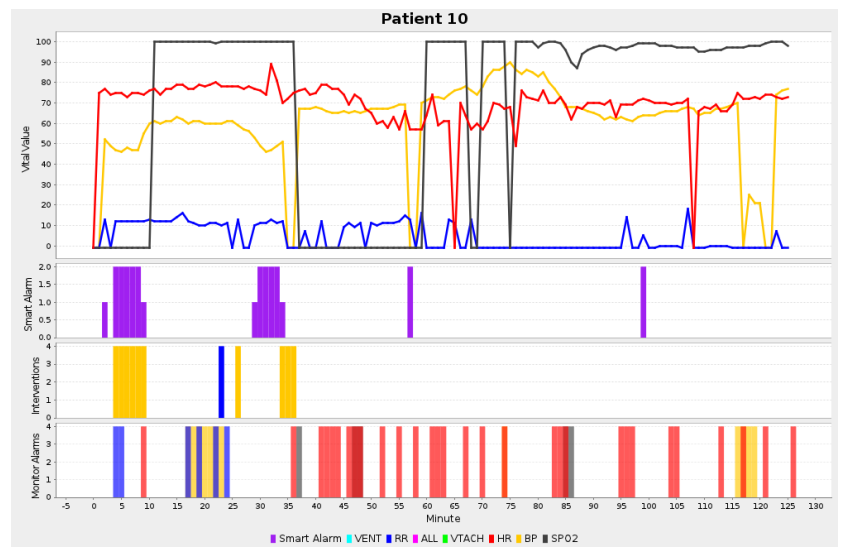

Fig. 3. Vitals signs and alarms; The top row is a trace of vital signs, smart alarm response is the second row, alarms resulting in an intervention are third from top and spurious alarms are in the bottom row. The smart alarm response tracks with the occurrence of intervention alarms.

alarm occured within 10 minutes of the intervention. Ten minutes would typically give clinicians time to react to an alarm. Figure 3 shows the patient vitals (top row), smart alarm output (second from top), non-intervention alarms (bottom row), and intervention alarms (third from top). For this observation, there were 4 discrete interventions, however those 4 events are covered by the two smart alarm events due to their temporal proximity.

The generated smart alarm output traces and observed alarm events were used to produce the follwing statistics for each observation:

1) Monitor Alarm Duration - The total amount of time monitor alarms (which did not lead to an intervention) were active during the observation.

2) Intervention Alarm Duration - The total amount of time monitor alarms (which did lead to an intervention) were active during the observation.

3) Smart Alarm Duration - The total amount of time the smart alarm was active during the observation.

4) Alarm Volume Ratio - The ratio of Smart Alarm Duration to Monitor Alarm Duration plus Intervention Alarm Duration. This statistic denotes the amount of time the smart alarm was active versus any monitor alarm.

5) False Negatives - The number of interventions resulting from alarms that were not covered by a smart alarm.

The effectiveness of the CABG-SA relative to the monitor alarms was measured via the Alarm Volume Ratio (a lower ratio indicates a more selective alarm) and the number of False Negatives. If the CABG-SA has 0 false negatives, then it is at least as safe as the existing monitor alarms.

\section{RESUlTS}

Table II contains the results of the analysis. Overall, the smart alarm produced fewer alarms. During study, the smart alarm was active $55 \%$ of the time that the standard monitor

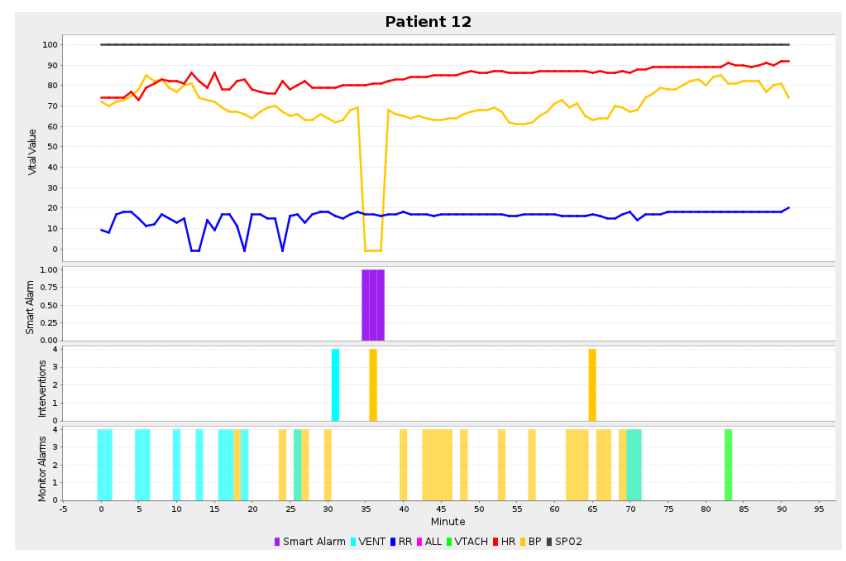

Fig. 4. Missed intervention

alarms were active. Of the 10 interventions, 9 were covered by the smart alarm.

\begin{tabular}{|c|c|c|c|c|c|c|c|}
\hline Patient & 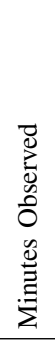 & 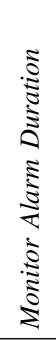 & 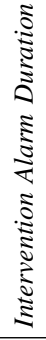 & 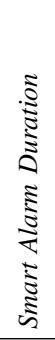 & 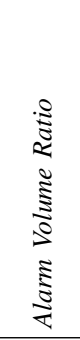 & 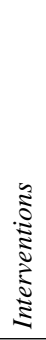 & 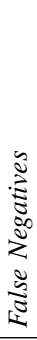 \\
\hline 8 & 46 & 16 & 0 & 44 & 2.750 & 0 & 0 \\
\hline 9 & 26 & 8 & 0 & 3.0 & 0.375 & 0 & 0 \\
\hline 10 & 127 & 7 & 43 & 15 & 0.300 & 4 & 0 \\
\hline 12 & 93 & 51 & 7 & 3 & 0.517 & 3 & 1 \\
\hline 13 & 62 & 29 & 2 & 4 & 0.130 & 3 & 0 \\
\hline 14 & 91 & 28 & 5 & 34 & 1.030 & 2 & 0 \\
\hline 17 & 122 & 33 & 3 & 11 & 0.310 & 1 & 0 \\
\hline 19 & 68 & 26 & 5 & 13 & 0.419 & 1 & 0 \\
\hline 22 & 116 & 38 & 0 & 40 & 1.053 & 0 & 0 \\
\hline aggregate & 751 & 236 & 65 & 167 & 0.55 & 13 & 1 \\
\hline
\end{tabular}

Smart Alarm vs. MONitor Alarm BEHAVIOR PER PATIENT

Many of the monitor alarms were spurious; Of the 301 minutes that alarms were active during observation, only 65 of those minutes were due to alarms that resulted in an intervention. In many cases, if a monitor alarm activated, a nurse would enter the room to either disable the alarm or recalibrate the alarm threshold.

Figure 4 shows the patient vitals aligned with the various alarms (both from the smart alarm and monitors) and the interventions. The first two interventions were covered by the smart alarm. The smart alarm failed to detect any phsyiologic instability that may have led to the last intervention.

\section{Evaluation, Discussion AND Future Work}

While this clinical evaluation demonstrates overall alarm reduction consistent with the earlier [6] evaluation (CABGSA fired approximately $55 \%$ of the time as the traditional alarms, see Figure 3 for an example of close to ideal behavior), CABG-SA did miss an important intervention (the 


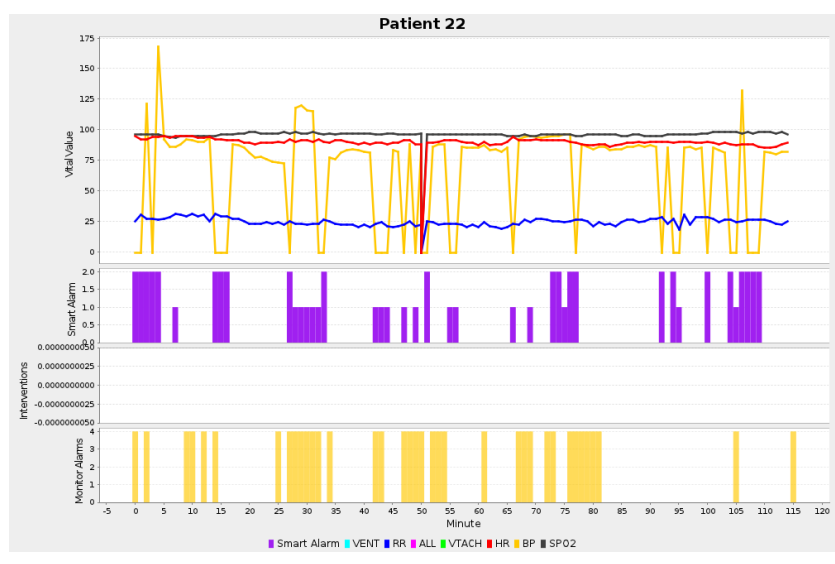

Fig. 5. CABG-SA false alarms

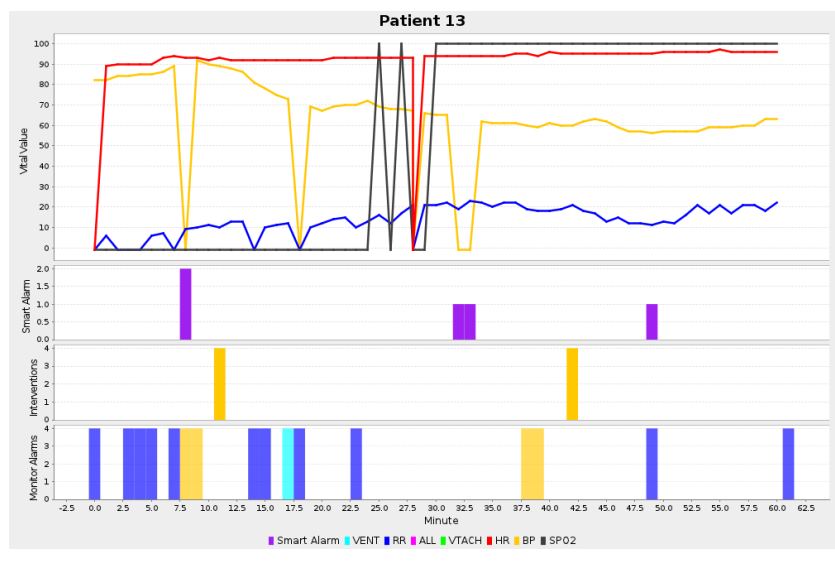

Fig. 6. Smart-Alarm due to dropped blood pressure signal

last intervention observed for Patient 12, see Figure 4). This particular intervention involved increasing the patient's dose of Neosenephrine (phenylephrine) which is used to increase blood pressure. Low blood pressure can be a problem in critical care, because low blood pressure can prevent the proper profusion of blood across vital organs, leading to organ failure. If the critical care staff were to miss an indication that they should perform an intervention to increase the patient's blood pressure, then that could have disastrous results.

Unfortunately, there isn't a rule that could be added to CABG-SA that would identify the last intervention without greatly increasing the sensitivity of the alarm. This is because during the time Patient 12 received the last intervention, Patient 12's vital signs would be classified as 'normal.' For the last intervention, the nurse was likely influenced by the downward trend of the patient's blood pressure. An improved version of CABG-SA should include rules for both the value of the physiologic state and the trending physiologic state.

Another interesting example is Patient 13 (Figure 6): the smart alarm which covers the first and second intervention was actually triggered by loss of the blood pressure signal (signified by an immediate drop of MAP to 0 ). If the blood pressure signal was not lost, the smart alarm would not have fired. Again, in this instance the blood pressure trend is what likely caused the nurse to intervene.

Patient 13 illustrates one way the method by which CABG-SA deals with missing or corrupted data is clearly not optimal. As described in Section II, CABG-SA interprets immediate drops to 0 as loss of signal and sets the corresponding set to 'Low.' This behavior is unsafe, as it may result in improper masking of serious physiologic instability. A more systematic approach of dealing with missing data is desirable. Previous work on open-loop safety for closed loop medical systems [9] leverage physiologic modeling to operate safely in an open loop situation (i.e. due to communications faults). A similar approach could be used to safely mask missing data while minimizing false alarms. For example, if CABG-SA loses the $\mathrm{SpO}_{2}$ signal at a particular moment in time, the algorithm could simulate (using a conservative physiologic model) the minimum amount of time, $t_{\min }$ it would take for the patient to fall past a danger threshold. CABG-GSA could then extrapolate the vital sign for $t_{\min }$ time units, then raise a false alarm. Thus CABGSA would mask any false alarms as long as possible without compromising safety.

\section{CONCLUSION}

Smarter clinical alarm systems will be necessary to help reduce the cognitive workload on critical care workers. In this paper we provide the results of a small clinical evaluation of CABG-SA, an alarm algorithm designed to leverage multiple vital signs to generate more specific alarms. The results of this study are consistent with previously reported alarm reduction statistics, however extra clinical information available in this study allowed us to more directly identify weaknesses of the algorithm and propose different ways CABG-SA could be extended to address those weaknesses.

\section{REFERENCES}

[1] J. Fairman and S. Kagan, "Creating critical care: the case of the Hospital of the University of Pennsylvania, 1950-1965," ANS Adv Nurs Sci, vol. 22, pp. 63-77, Sep 1999.

[2] G. B. Hannibal, "Monitor alarms and alarm fatigue," AACN Adv Crit Care, vol. 22, pp. 418-420, 2011.

[3] S. Siebig, L. Juhls, M. Imhoff, U. Gather, U. Scholmerich, and C. Wrede, "Plug-and-play for medical devices: Experiences from a case study," Crit Care Med, vol. 38, no. 2, pp. 451-455, 2010.

[4] K. C. Graham and M. Cvach, "Monitor alarm fatigue: standardizing use of physiological monitors and decreasing nuisance alarms," Am. $J$. Crit. Care, vol. 19, pp. 28-34, Jan 2010.

[5] M. Mullen-Fortino and N. O'Brien, "Caring for a patient after coronary artery bypass graft surgery," Nursing, vol. 38, no. 3, pp. 46-52, March 2008.

[6] A. L. King, A. Roederer, D. Arney, S. Chen, M. Mullen-Fortino, A. Giannareas, W. H. III, V. Kern, N. Stevens, J. Tannen, A. V. Trevino, S. Park, O. Sokolsky, and I. Lee, "Gsa: a framework for rapid prototyping of smart alarm systems," in IHI, 2010, pp. 487-491.

[7] "Physiobank medical device data stream repository."

[8] "Phillips VisiCUE," http://www.healthcare.philips.com/main/products/ patient_monitoring/products/eicu/index.wpd, 2010.

[9] D. Arney, M. Pajic, J. M. Goldman, I. Lee, R. Mangharam, and O. Sokolsky, "Toward patient safety in closed-loop medical device systems," in Proceedings of the 1st ACM/IEEE International Conference on Cyber-Physical Systems, ser. ICCPS '10. New York, NY, USA: ACM, 2010, pp. 139-148. [Online]. Available: http://doi.acm.org/10.1145/1795194.1795214 